\title{
Coronary artery disease is associated with an increased mortality rate following video-assisted thoracoscopic lobectomy
}

\author{
Alberto Sandri, MD, ${ }^{\mathrm{a}}$ Rene Horsleben Petersen, MD, ${ }^{\mathrm{b}}$ Herbert Decaluwé, MD,${ }^{\mathrm{c}}$ Johnny Moons, CCRC ${ }^{\mathrm{c}}$ \\ Mark K. Ferguson, MD, ${ }^{\mathrm{d}}$ Henrik Jessen Hansen, $\mathrm{MD},{ }^{\mathrm{b}}$ and Alessandro Brunelli, $\mathrm{MD}^{\mathrm{a}}$
}

\begin{abstract}
Objective: To compare the incidence of major adverse cardiac events (MACE) and mortality following video-assisted thoracoscopic surgery (VATS) lobectomy in patients with and without coronary artery disease (CAD).

Methods: Multicentre retrospective analysis of 1699 patients undergoing VATS lobectomy (January 2012-March 2015). CAD definition: previous acute myocardial infarct (AMI), angina, percutaneous coronary intervention (PCI) or coronary artery bypass graft (CABG). MACE definition: postoperative acute myocardial ischemia, cardiac arrest or any cardiac death. Propensity score analysis was performed to match patients with and without CAD. Outcomes of the 2 matched groups were compared.
\end{abstract}

Results: The incidence of MACE and mortality for the entire population was $0.4 \%$ (7 patients) and 1.7\% (29 patients); 218 patients (13\%) had a history of CAD: 106 previous AMI, 55 angina, $32 \mathrm{CABG}$, and $81 \mathrm{PCI}$. The propensity score yielded 2 well-balanced groups of 218 pairs with and without CAD. MACE (CAD 2 $[0.9 \%]$ vs no-CAD $1[0.5 \%] ; P=1)$, cardiovascular and pulmonary complications (CAD 61 [28\%] vs no-CAD 51 [23\%]; $P=.3$ ) and postoperative stay (CAD 7.3 days vs no-CAD 6.2 days; $P=.3$ ) were not different between the groups. The incidence of atrial fibrillation (CAD 31 [14\%] vs no-CAD 18 [8.2\%]; $P=.07)$, 30-day mortality (CAD: $11[5 \%]$ vs no-CAD $2[0.9 \%] ; P=.02)$ and death among complicated patients (CAD 18\% vs no-CAD $3.9 \% ; P=.009$ ) were higher in the CAD group.

Conclusions: The incidence of MACE following VATS lobectomy in patients with CAD is low and similar to patients without CAD. However, their risk of postoperative mortality is fivefold higher compared with non-CAD patients, warranting refined preoperative functional evaluation and more intense postoperative monitoring. (J Thorac Cardiovasc Surg 2017;154:352-7)



Patients with CAD had higher 30-day mortality than those without CAD after VATS lobectomy.

\section{Central Message \\ Coronary artery disease is associated with increased mortality after VATS lobectomy. This information is important to inform the shared decision-making process and to opti- mize patient care.}

\section{Perspective}

Coronary artery disease is common in candidates for lung cancer resection. There is scant evidence regarding its impact on the outcome of VATS lobectomy. We found that patients with CAD had few postoperative major adverse cardiac events but fivefold higher 30-day mortality compared with non-CAD patients. This information is important to inform the shared decision-making process and optimize patient care.

See Editorial Commentary page 358.
Coronary artery disease (CAD) is frequently encountered in candidates for lung cancer surgery. Recent data from large organizational databases show that this condition is present

\footnotetext{
From the a Department of Thoracic Surgery, St James's University Hospital, Leeds, United Kingdom; ' Department of Cardio-thoracic Surgery, Copenhagen University Hospital, Copenhagen, Denmark; ${ }^{\mathrm{c}}$ Department of Thoracic Surgery UZ Leuven, Leuven, Belgium; and ${ }^{\mathrm{d}}$ Department of Surgery and The Comprehensive Cancer Center, The University of Chicago, Chicago, Ill.

Received for publication Nov 9, 2016; revisions received Feb 19, 2017; accepted for publication March 5, 2017; available ahead of print April 12, 2017.

Address for reprints: Alessandro Brunelli, MD, Department of Thoracic Surgery, St James's University Hospital Bexley Wing, Beckett St, Leeds LS9 7TF, United Kingdom (E-mail: alexit_2000@yahoo.com).

$0022-5223 / \$ 36.00$

Copyright (c) 2017 by The American Association for Thoracic Surgery

http://dx.doi.org/10.1016/j.jtcvs.2017.03.042
}

in $7 \%$ to $16 \%$ of patients affected by resectable lung cancer. $^{1,2}$

The risk of major adverse cardiac events (MACE) following anatomic lung resection for lung cancer is approximately $3 \% .^{3}$ However, the literature available on cardiac risk and lung cancer surgery is scarce, particularly regarding the impact of this comorbidity on outcomes following

Scanning this QR code will take you to a supplemental video for the article. 


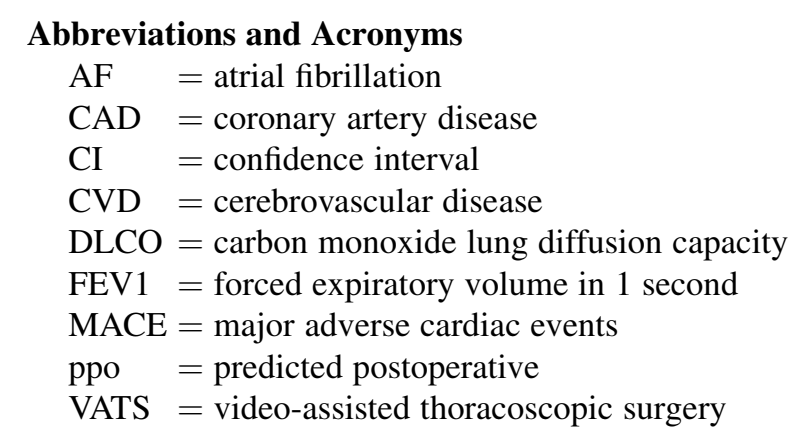

video-assisted thoracoscopic surgery (VATS) lobectomy, which has become the treatment of choice for early-stage non-small-cell lung cancer in many centers. ${ }^{4,5}$ Compared with patients undergoing lobectomy through thoracotomy, those operated through VATS have reduced incidence of complications (including cardiovascular complications) and in-hospital mortality. The benefits of VATS have been shown to be particularly evident in high-risk patients. ${ }^{6-10}$ For this reason an increasing number of patients with underlying comorbidities and traditionally considered at high risk for surgery are now considered surgical candidates. Therefore, we felt that there was a need to fill the knowledge gap about the influence of CAD on the outcome of patients undergoing VATS lobectomy.

The objective of this study was to assess the influence of $\mathrm{CAD}$ on the outcome of patients undergoing VATS lobectomy for lung cancer.

\section{PATIENTS AND METHODS}

We involved 4 thoracic surgery units ( 3 from Europe and 1 from the United States) and performed a retrospective analysis on prospectively collected data of patients submitted to VATS lobectomy (January 2012March 2015) for lung cancer. Patients whose operations started with a VATS approach and then were converted to open surgery for technical reasons or major intraoperative complications were also included in this analysis ( 88 patients). We chose to include the converted patients as we believe analyses about VATS anatomic resections should be performed as an intention-to-treat one. The inclusion of patients who required conversion to open approach ensure a more realistic outcome analysis. ${ }^{11}$ The local institution review boards reviewed the study, which was classified as service evaluation not requiring ethics committee approval.

Surgical treatment was discussed and agreed by the local multidisciplinary tumor boards. Patients were referred to a specialist cardiologist for noninvasive testing and to optimize their medical treatment in case their cardiac risk was considered high (ie, cardiac risk index $>2$ ) and according to current guidelines. ${ }^{12,13}$ No patients in this series had a prophylactic revascularization procedure before the operation. All patients submitted to lung resections were deemed hemodynamically stable.

Inoperability criteria included a predicted postoperative forced expiratory volume in 1 second (ppoFEV1) or a predicted postoperative carbon monoxide lung diffusing capacity (ppoDLCO) $<30 \%$, in association with a maximum oxygen consumption $\left(\mathrm{VO}_{2} \max \right)<10 \mathrm{~mL} / \mathrm{kg} / \mathrm{min}$, whenever performed. ${ }^{13}$

All patients were operated on by board-qualified thoracic surgeons through a 2- to 3-port VATS anterior approach, as per surgeon preference. Taking into account the differences of practice among the participating centers, patients were managed according to similar standardized pathways of care pre- and postoperatively, including early mobilization, venous thromboembolism prophylaxis, chest physiotherapy, and physical rehabilitation. Surgical pain control was achieved in most of the patients by using a combination of paravertebral analgesia with local anaesthetics and intravenous patient-controlled analgesia.

\section{Definitions}

For the purpose of this study, CAD was defined as follows: previous acute myocardial infarct (AMI), angina, previous percutaneous coronary intervention $(\mathrm{PCI})$, and/or previous coronary artery bypass graft (CABG). MACE included postoperative acute myocardial ischemia (evidenced by the appearance of a new $\mathrm{Q}$ wave in 2 or more contiguous leads on electrocardiogram or by laboratory isoenzyme evidence of myocardial necrosis), ${ }^{14}$ cardiac arrest, and any cardiac death. Definitions of CAD and MACE were discussed and agreed among the coinvestigators before data from the individual centers were merged into a common dataset for the analysis. All these variables were elements already present in the prospectively maintained institutional databases of each unit. Discrepancies or incomplete information were verified by review of the medical records performed by the local investigators at each center.

Cardiovascular and pulmonary complications were defined as those occurring in-hospital or within 30 days from operation, and included acute respiratory distress syndrome, atrial arrhythmia, ventricular arrhythmia, bronchoscopy for atelectasis, pneumonia, pulmonary embolism, deep vein thrombosis, myocardial infarct, acute renal failure, stroke. Their definitions were in accordance with the Society of Thoracic Surgeons and European Society of Thoracic Surgeons joint standardization of variables. ${ }^{14}$ Mortality was defined as any death occurring within 30 days or over a longer period if the patient was still admitted in the hospital.

For the purpose of this study, failure-to-rescue was defined as any death (in-hospital or within 30 days) that occurred in patients who developed cardiovascular or pulmonary complications according to the previous definition (irrespective of the severity of complications).

\section{Statistical Methods}

To minimize the impact of other confounders on the outcome, patients with and without CAD were compared after matching by using a propensity score case matching analysis. ${ }^{15}$ Propensity scores were estimated by logistic regression analysis including baseline and surgical characteristics: age, sex, FEV1\%, DLCO $\%$, history of cerebrovascular disease, diabetes, neoadjuvant chemotherapy, side and site of lobectomy, conversion to thoracotomy. Clustering of data at the institutional level was taken into account by using a panel data analysis with the variable "Institution" as a cluster variable. All variables except DLCO (5\% missing data) were complete and there was no missing endpoint variable. Missing variables were imputed by averaging the nonmissing values (numeric variables)

Patients with and without CAD were then matched 1:1 according to their nearest propensity score without replacement. The propensity score analysis yielded 2 groups of matched pairs that were then compared in terms of baseline characteristics and outcome.

To evaluate the magnitude of the differences of the variables between the 2 groups, we used the standardized difference (effect size). Effect size is calculated by dividing the difference of the averages of the 2 groups by the standard deviation of the total population. An effect size lower than 0.2 indicates a small difference. The standardized difference is considered more appropriate than $P$ value to assess the adequacy of the matching process, as it is less affected by sample size. ${ }^{16}$ The incidences of MACE and mortality were compared by means of the Fisher exact test. A $P$ value of .05 was accepted as significant. In addition, as a further method of risk adjustment, we included the propensity score in a multivariable comparison of outcome along with the comparison variable of interest $(\mathrm{CAD}) .{ }^{15,17}$ 
All tests were 2-tailed and a significance level of .05 was considered. The statistical tests were performed on the statistical software Stata 12.0 (Stata Corp, College Station, TX).

\section{RESULTS}

The baseline characteristics of all 1699 patients included in the study are described in Table 1. Of these patients, 218 $(13 \%)$ had a history of CAD: 106 previous AMI, 55 angina, $32 \mathrm{CABG}$, and $81 \mathrm{PCI}$ with some patients who experienced more than 1 event. MACE and mortality rates for the entire population were $0.4 \%$ ( 7 patients) and $1.7 \%$ (29 patients), respectively.

The most frequent cause of death was respiratory (15 cases). Four patients died of pulmonary embolism, 5 of sepsis, and 2 of multiorgan failure. Only 3 patients experienced cardiac-related deaths.

The unmatched comparison between patients with and without CAD is shown in Table 2. In summary, compared with patients without $\mathrm{CAD}$, those with $\mathrm{CAD}$ were older, more frequently men, had a lower DLCO, and had a higher incidence of previous cerebrovascular disease (CVD) and diabetes (effect sizes greater than 0.2).

The propensity score matching yielded 2 groups of 218 pairs of patients with and without CAD, well balanced for baseline characteristics (Table 3).

Table 4 shows the results of the comparison between the 2 matched groups. Briefly, the incidence of MACE $(P=1)$, the rate of cardiovascular and pulmonary complications $(P=.3)$, and the postoperative stay $(P=.3)$ were not different between the 2 matched groups. Compared with patients without CAD, those with CAD experienced a higher rate of atrial fibrillation (AF) $(P=.07)$ and fivefold higher 30 -day mortality rate $(P=.02)$ (Figure 1$)$. When the propensity score was used to adjust the risk in a multivariable regression analysis performed on all patients (matched and unmatched), CAD was not significantly associated with MACE (regression coefficient 0.5, SE 0.9, $P=.6$ ). However, CAD remained significantly associated with

TABLE 1. Characteristics of the patients included in the study (1699 patients)

\begin{tabular}{lc}
\hline \multicolumn{1}{c}{ Variables } \\
\hline Age & $67.4(61-74)$ \\
Sex male, $\mathrm{n}(\%)$ & $791(47 \%)$ \\
FEV1\% & $86.8(73-99)$ \\
DLCO $\%$ & $72(62-80)$ \\
CVD, $\mathrm{n}(\%)$ & $95(5.6)$ \\
Diabetes, $\mathrm{n}(\%)$ & $159(9.2)$ \\
Side right, $\mathrm{n}(\%)$ & $995(59)$ \\
Site upper, $\mathrm{n}(\%)$ & $888(52)$ \\
\hline Results are expressed as medians and interquartile ranges (numeric variables) or as \\
number and percentages (categorical variables). FEVI, Forced expiratory volume \\
in 1 second; $D L C O$, carbon monoxide lung diffusion capacity; CVD, cerebrovascular \\
disease.
\end{tabular}

TABLE 2. Unmatched comparison of baseline characteristics between patients with and without CAD

\begin{tabular}{lccc}
\hline \multicolumn{1}{c}{ Variables } & $\begin{array}{c}\text { Patients with } \\
\text { CAD (218) }\end{array}$ & $\begin{array}{c}\text { Patients without } \\
\text { CAD (1481) }\end{array}$ & $\begin{array}{c}\text { Effect } \\
\text { size }\end{array}$ \\
\hline Age & $70.75(6.8)$ & $65.85(10.7)$ & 0.47 \\
Sex male, n (\%) & $138(63)$ & $653(44)$ & 0.39 \\
FEV1\% & $84.13(18.8)$ & $87.14(20.4)$ & 0.15 \\
DLCO\% & $68.74(18.5)$ & $72.65(18.9)$ & 0.24 \\
CVD, n (\%) & $22(10)$ & $73(4.9)$ & 0.23 \\
Diabetes, n (\%) & $39(18)$ & $120(8.1)$ & 0.34 \\
Neoadjuvant & $9(0.40)$ & $57(0.38)$ & 0.01 \\
$\quad$ chemotherapy, n (\%) & & & \\
Side right, n (\%) & $145(67)$ & $850(57)$ & 0.19 \\
Site upper, n (\%) & $122(56)$ & $766(52)$ & 0.08 \\
\hline Re
\end{tabular}

Results are expressed as means and standard deviations (numeric variables) or as number and percentages (categorical variables). Effect size or standardized difference is calculated by dividing the difference of the averages of the 2 groups by the standard deviation in the total population. Effect size: 0.2 small difference; 0.5 medium difference; 0.8 large difference. $C A D$, Coronary artery disease; $F E V I$, forced expiratory volume in 1 second; $D L C O$, carbon monoxide lung diffusion capacity; $C V D$, cerebrovascular disease.

30-day mortality (regression coefficient 0.9 , SE 0.4, odds ratio $2.5, P=.03$ ).

The failure-to-rescue rate in the matched groups (the mortality rate observed among patients with postoperative cardiovascular and pulmonary complications) was higher in the patients with CAD compared with those without CAD (CAD: 18\%, 95\% confidence interval [CI] 9.4-30.0 vs no-CAD: $3.9 \%, 95 \%$ CI $0.5-13.0, P=.009$ ).

Of the 88 VATS lobectomies that were converted to thoracotomy, 16 had CAD and their mortality rate was not statistically significantly different than in those non-CAD patients who were converted $(13 \%, 95 \%$ CI $2-38$ vs $7 \%$, $95 \%$ CI 2-15, $P=.6$ ) (Video 1).

\section{DISCUSSION}

\section{Background and Rationale for the Study}

$\mathrm{CAD}$ is a frequent comorbidity in candidates to lung resection. The incidence of $\mathrm{CAD}$ in patients with operable lung cancer has been reported to vary from $8 \%$ to $16 \% .^{1,2}$ The presence of CAD has been associated with increased morbidity and mortality following lung resection. ${ }^{1,18-23}$ In some of these studies, CAD was even more important than pulmonary function as a risk factor for postoperative major morbidity, increasing the risk by 1.6 times. ${ }^{21}$ Nevertheless, the impact of CAD in a selected population of patients with lung cancer operated on by VATS has never been reported. The rationale for investigating the effect of CAD in this group of patients is to provide information to be used during multidisciplinary discussion for selecting the best treatment option and to be shared with the patient at the time of surgical consultation. In fact, the use of VATS has become the recommended approach for early-stage lung cancer radical treatment ${ }^{5}$ and 
TABLE 3. Comparison of baseline characteristics between matched groups of patients with and without CAD (218 matched pairs)

\begin{tabular}{lccc}
\hline \multicolumn{1}{c}{ Variables } & $\begin{array}{c}\text { Patients } \\
\text { with CAD }\end{array}$ & $\begin{array}{c}\text { Patients } \\
\text { without CAD }\end{array}$ & $\begin{array}{c}\text { Effect } \\
\text { size }\end{array}$ \\
\hline Age & $70.75(6.8)$ & $71.07(7.2)$ & 0.05 \\
Sex male, n (\%) & $138(63)$ & $129(59)$ & 0.08 \\
FEV1\% & $84.13(18.8)$ & $83.01(20.9)$ & 0.06 \\
DLCO\% & $68.74(18.5)$ & $68.08(17.5)$ & 0.04 \\
CVD, n (\%) & $22(10)$ & $26(12)$ & 0.07 \\
Diabetes, n (\%) & $39(18)$ & $41(19)$ & 0.03 \\
Neoadjuvant & $9(4.0)$ & $7(3.2)$ & 0.05 \\
$\quad$ chemotherapy, n (\%) & & & \\
Side right, n (\%) & $145(67)$ & $134(62)$ & 0.10 \\
Site upper, n (\%) & $122(56)$ & $124(57)$ & 0.02 \\
\hline
\end{tabular}

Results are expressed as means and standard deviations (numeric variables) or as number and percentages (categorical variables). Effect size or standardized difference is calculated by dividing the difference of the averages of the 2 groups by the standard deviation in the total population. Effect size: 0.2 small difference; 0.5 medium difference; 0.8 large difference. $C A D$, Coronary artery disease; $F E V I$, forced expiratory volume in 1 second; $D L C O$, carbon monoxide lung diffusion capacity; $C V D$, cerebrovascular disease.

has challenged traditional operability criteria, having shown its beneficial effects particularly in high-risk patients. ${ }^{6,9}$ This has allowed operating on an increasing number of patients with underlying comorbidities, including cardiovascular comorbidities.

For this reason, we wanted to verify the incidence of major cardiac events and mortality in patients with and without CAD undergoing VATS lobectomy.

\section{Main Findings}

We found that patients with CAD had a similar and very small incidence of MACE compared with a matched cohort of patients without CAD. A previous study including mostly thoracotomy patients found that the incidence of MACE in patients with $\mathrm{CAD}$ was approximately $6 \%$ compared with only $1 \%$ in those without CAD. ${ }^{22}$ Similarly, Fernandez and colleagues, ${ }^{23}$ using the Surveillance, Epidemiology, and End Results-Medicare database, found that patients with a previous coronary stent procedure within 1 year from lung cancer resection had postoperative MACE and mortality rates of $9.3 \%$ and $7.7 \%$, respectively. Although the surgical access was not specified, it seems plausible that most patients in the latter study were operated on through a thoracotomy, as the series spanned back to 1998 .

In our series, the rate of MACE in patients with CAD was only $0.9 \%$ (vs $0.5 \%$ in non-CAD patients). This low incidence of MACE likely reflects a protective effect of VATS in this high-risk group of patients.

We also found that in spite of a similar incidence of overall cardiovascular and pulmonary complications, patients with CAD had a fivefold higher risk of 30-day mortality compared with their matched counterparts without CAD. This discrepant result is explained in part by the higher incidence of patients who died after developing complications in the CAD group compared with the non-CAD group. The failure-to-rescue rate in the matched population was almost fivefold higher among complicated patients with CAD compared with complicated patients without CAD.

One possible explanation can be found in the reduced aerobic reserve of patients with CAD, caused by a direct effect of the underlying cardiac disease or indirectly by physical deconditioning. The occurrence of a postoperative complication in this situation may increase the oxygen demand to a level exceeding the aerobic capacity of the patient leading in turn to an anaerobic metabolism, lactic acidemia, multiorgan failure, and eventually death. In this regard, CAD is not the direct cause of death, as shown by the low incidence of cardiac deaths in this series, but a predisposing factor exposing the patient to a higher risk in case of postoperative complications.

Unfortunately, details about baseline cardiac function (ie, ejection fraction) or maximum oxygen consumption during cardiopulmonary exercise test were not available in most of the patients as per institutional preoperative evaluation pathways. They would have certainly added useful information to this study to clarify the cause of the increased mortality among complicated patients with CAD. Certainly, these results warrant future prospective investigations aimed at validating this hypothesis.

Another interesting finding of our analysis was regarding the incidence of AF. Although the incidence of AF in patients without $\mathrm{CAD}(7 \%)$ was in line with the incidence reported in other large VATS series, ${ }^{2,10}$ AF was twice as common in patients with CAD than in patients without it.

TABLE 4. Comparison of outcomes between matched groups of patients with and without CAD (218 pairs)

\begin{tabular}{|c|c|c|c|}
\hline & Patients with CAD $(n=218)$ & Patients without CAD $(n=218)$ & $\boldsymbol{P}$ \\
\hline MACE, n (\%) & $2(0.9 \%, 0.1-3.2)$ & $1(0.5 \%, 0.1-2.5)$ & 1 \\
\hline Cardiopulmonary complications, $\mathrm{n}(\%)$ & $61(28,22-34)$ & $51(23,18-30)$ & .3 \\
\hline LOS, d & $7.3(6.3-8.3)$ & $6.2(5.5-6.9)$ & .3 \\
\hline $\mathrm{AF}, \mathrm{n}(\%)$ & $31(14,9.9-19.0)$ & $18(8.2,5-13)$ & .07 \\
\hline 30-d mortality, n $(\%)$ & $11(5.0,2.5-8.8)$ & $2(0.9,0.1-3.2)$ & .02 \\
\hline
\end{tabular}

Results are expressed as means and $95 \%$ confidence intervals (numeric variables) or numbers, percentages and $95 \%$ confidence intervals (categorical variables). CAD, Coronary artery disease; $M A C E$, major adverse cardiac events; $L O S$, length of postoperative stay; $A F$, atrial fibrillation. 


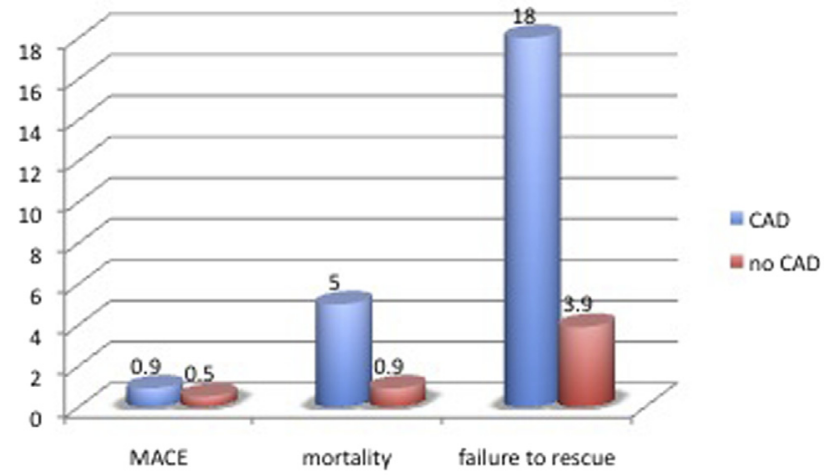

FIGURE 1. Comparison of outcomes between matched patients with and without coronary artery disease $(C A D)$. MACE, Major adverse cardiac events.

A previous study showed that CAD (previous percutaneous transluminal coronary angioplasty, stent placement, and preexisting history of angina) was an independent risk factor for $\mathrm{AF}$, with a fourfold increased risk compared with patients without CAD. ${ }^{24}$ However, other studies did not find $\mathrm{CAD}$ as a risk factor associated with $\mathrm{AF}$ after adjusting the analysis for other confounders. ${ }^{25,26}$

\section{Study Limitations}

This study has potential limitations. First, this is a retrospective analysis on prospectively collected data from 4 different centers. Inherent selection bias may be present, as in all retrospective analyses of this kind. However, to minimize this problem, we used a propensity score analysis, which is considered the most reliable method for a balanced comparison in a nonrandomized setting. ${ }^{15}$ However, propensity score analysis does not take into account unmeasured or unknown factors associated with outcome. Moreover, it does not completely adjust accurately for biases resulting from cohort selection. In addition, the construction of the propensity score and the adequate balancing of groups were limited by the variables available and the small sample size. For instance, important variables that could have been associated with the outcome of interest, such as smoking status or clinical stage, were not available for all patients and were not used in the analysis.

In addition, owing to the nature of study, information about the patients with CAD at risk of having surgery who were initially referred to surgeons and then underwent nonsurgical treatment was not available. As mentioned previously, this introduced an inherent selection bias that was difficult to control completely even using adequate balancing with propensity score.

Second, details about cardiac function were not available in most patients. This information would have been useful to clarify the causal relationship between incidence of complications and mortality in patients with CAD. Similarly, a
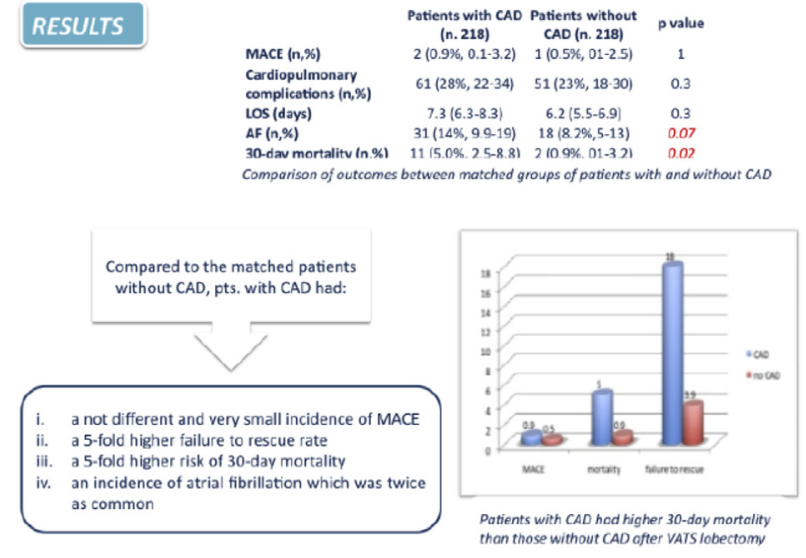

VIDEO 1. Dr Alberto Sandri describes on behalf of his coauthors the rationale, objective, methods, and main findings of this study. Video available at: http://www.jtcvsonline.org/article/S0022-5223(17)30546-9/ addons.

cardiopulmonary exercise test was performed in a small number of patients due to institutional protocols. Therefore, objective data about aerobic capacity were missing in this series.

Third, for the retrospective nature of the study, information about medical treatment of CAD at the time of surgery was not available. Likewise, the precise number of patients who were referred to a cardiologist before surgery for optimization of their medical treatment or additional testing was not available in this dataset. Nevertheless, as mentioned previously, none of the patients in this series received a prophylactic invasive cardiologic procedure (ie, PCI or stent) before lung resection.

Finally, the low incidence of MACE has limited the statistical power of the study to detect any significant difference between CAD and non-CAD groups. The calculated power for the comparison of the 2 matched groups to detect a significant difference with an alpha level of 0.05 in our population was only $14 \%$. With such a low rate of MACE, a 30-fold larger sample size would be needed to have a statistical power of at least $80 \%$. Future large data analyses would be warranted to confirm our results.

\section{CLINICAL IMPLICATIONS AND CONCLUSIONS}

We showed that, despite a very low incidence of MACE, the presence of CAD is associated with a fivefold higher risk of mortality after VATS lobectomy. Based on this finding, we have started to refer patients with a history of $\mathrm{CAD}$ to cardiopulmonary exercise test to better evaluate the functional reserve and to identify deficits in the oxygen transport system to be possibly corrected before the operation. Moreover, our results represent useful information to be used to decide the best treatment strategy for this highrisk group of patients during multidisciplinary meetings. Finally, taking into the account the several limitations 
discussed previously, the findings of our study can be used as information to be shared with the patients during the surgical consultation.

\section{Conflict of Interest Statement}

Dr Brunelli reports speaker honoraria from BARD. Dr Petersen reports personal fees from Medtronic, Ethicon, and Medela. Dr Hansen reports personal fees from the speakers bureau of Medtronic and BARD. All other authors have nothing to disclose with regard to commercial support.

\section{References}

1. Brunelli A, Salati M, Rocco G, Varela G, Van Raemdonck D, Decaluwe H, et al. European risk models for morbidity (EuroLung1) and mortality (EuroLung2) to predict outcome following anatomic lung resections: an analysis from the European Society of Thoracic Surgeons database. Eur J Cardiothorac Surg. 2017;51: 490-7.

2. Paul S, Altorki NK, Sheng S, Lee PC, Harpole DH, Onaitis MW, et al. Thoracoscopic lobectomy is associated with lower morbidity than open lobectomy: a propensity-matched analysis from the STS database. J Thorac Cardiovasc Surg. 2010;139:366-78.

3. Brunelli A, Varela G, Salati M, Jimenez MF, Pompili C, Novoa A, et al. Recalibration of the revised cardiac risk index in lung resection candidates. Ann Thorac Surg. 2010;90:199-203.

4. Yan TD, Cao C, D’Amico TA, Demmy TL, He J, Hansen H, et al. Video-assisted thoracoscopic surgery lobectomy at 20 years: a consensus statement. Eur J Cardiothorac Surg. 2014;45:633-9.

5. Howington JA, Blum MG, Chang AC, Balekian AA, Murthy SC. Treatment of stage I and II non-small cell lung cancer: diagnosis and management of lung cancer, 3rd ed: American College of Chest Physicians evidence-based clinical practice guidelines. Chest. 2013;143:e278S-313.

6. Sandri A, Papagiannopoulos K, Milton R, Chaudhuri N, Kefaloyannis E, Pompili C, et al. High-risk patients and postoperative complications following video-assisted thoracic surgery lobectomy: a case-matched comparison with lower-risk counterparts. Interact Cardiovasc Thorac Surg. 2015;21:761-5.

7. Zhang R, Ferguson MK. Video-assisted versus open lobectomy in patients with compromised lung function: a literature review and meta-analysis. PLoS One. 2015; 10:e0124512.

8. Demmy TL, Curtis JJ. Minimally invasive lobectomy directed toward frail and high-risk patients: a case-control study. Ann Thorac Surg. 1999;68:194-200.

9. Burt BM, Kosinski AS, Shrager JB, Onaitis MW, Weigel T. Thoracoscopic lobectomy is associated with acceptable morbidity and mortality in patients with predicted postoperative forced expiratory volume in 1 second or diffusing capacity for carbon monoxide less than $40 \%$ of normal. J Thorac Cardiovasc Surg. 2014; 148:19-28.

10. Falcoz PE, Puyraveau M, Thomas PA, Decaluwe H, Hürtgen M, Petersen RH, et al. Video-assisted thoracoscopic surgery versus open lobectomy for primary non-small-cell lung cancer: a propensity-matched analysis of outcome from the European Society of Thoracic Surgeon database. Eur J Cardiothorac Surg. 2016;49:602-9.

11. Brunelli A. Video-assisted thoracoscopic lobectomy: intention-to-treat analysis to convey realistic outcomes. Eur J Cardiothorac Surg. 2016;49:876.
12. Fleisher LA, Beckman JA, Brown KA, Calkins H, Chaikof EL, Fleischmann KE et al. ACC/AHA 2007 guidelines on perioperative cardiovascular evaluation and care for noncardiac surgery: a report of the American College of Cardiology/ American Heart Association Task Force on Practice Guidelines (Writing Committee to Revise the 2002 Guidelines on Perioperative Cardiovascular Evaluation for Noncardiac Surgery): developed in collaboration with the American Society of Echocardiography, American Society of Nuclear Cardiology, Heart Rhythm Society, Society of Cardiovascular Anesthesiologists, Society for Cardiovascular Angiography and Interventions, Society for Vascular Medicine and Biology, and Society for Vascular Surgery. Circulation. 2007;116:e418-99.

13. Brunelli A, Kim AW, Berger KI, Addrizzo-Harris DJ. Physiologic evaluation of the patient with lung cancer being considered for resectional surgery. Chest 2013;143:166S-90S.

14. Fernandez FG, Falcoz PE, Kozower BD, Salati M, Wright CD, Brunelli A. The Society of Thoracic Surgeons and the European Society of Thoracic Surgeons general thoracic surgery databases: joint standardization of variable definitions and terminology. Ann Thorac Surg. 2015;99:368-76.

15. Blackstone EH. Comparing apples and oranges. J Thorac Cardiovasc Surg. 2002; $123: 8-15$.

16. Austin PC, Mamdani MM. A comparison of propensity score methods: a casestudy estimating the effectiveness of post-AMI statin use. Stat Med. 2006;25: 2084-106.

17. Cook EF, Goldman L. Performance of tests of significance based on stratification by a multivariate confounder score or by a propensity score. J Clin Epidemiol. 1989;42:317-24.

18. Ambrogi V, Pompeo E, Elia S, Pistolese GR. MineoTC. The impact of cardiovascular comorbidity on the outcome of surgery for stage I and II non-small-cell lung cancer. Eur J Cardiothorac Surg. 2003;23:811-7.

19. Ferguson MK, Saha-Chaudhuri P, Mitchell JD, Varela G, Brunelli A. Prediction of major cardiovascular events after lung resection using a modified scoring system. Ann Thorac Surg. 2014;97:1135-40.

20. Ciriaco P, Carretta A, Calori G, Mazzone P, Zannini P. Lung resection for cancer in patients with coronary arterial disease: analysis of short-term results. Eur J Cardiothorac Surg. 2002;22:35-40.

21. Puri V, Crabtree TD, Bell JM, Kreisel D, Krupnick AS, Broderick S, et al. National cooperative group trials of "high-risk" patients with lung cancer: are they truly "high-risk"? Ann Thorac Surg. 2014;97:1678-83.

22. Ferguson MK, Celauro AD, Vigneswaran WT. Validation of a modified scoring system for cardiovascular risk associated with major lung resection. Eur J Cardiothorac Surg. 2012;41:598-602.

23. Fernandez FG, Crabtree TD, Liu J, Meyers BF. Incremental risk of prior coronary arterial stents for pulmonary resection. Ann Thorac Surg. 2013;95:1212-8.

24. Ivanovic J, Maziak DE, Ramzan S, McGuire AL, Villeneuve PJ, Gilbert S, et al Incidence, severity and perioperative risk factors for atrial fibrillation following pulmonary resection. Interact Cardiovasc Thorac Surg. 2014;18:340-6.

25. Vaporciyan A, Correa AM, Rice DC, Roth JA, Smythe WR, Swisher S, et al. Risk factors associated with atrial fibrillation after noncardiac thoracic surgery: analysis of 2588 patients. J Thorac Cardiovasc Surg. 2004;127:779-86.

26. Onaitis M, D'Amico T, Zhao Y, O'Brien S, Harpole D. Risk factors for atrial fibrillation after lung cancer surgery: analysis of The Society of Thoracic Surgeons General Thoracic Surgery Database. Ann Thorac Surg. 2010;90:368-74.

Key Words: lung cancer, lung resection, coronary artery disease, video-assisted thoracoscopic surgery, mortality, morbidity 